Check for updates

Cite this: RSC Adv., 2017, 7, 56306

Received 23rd October 2017

Accepted 2nd December 2017

DOI: $10.1039 / c 7 r a 11711 f$

rsc.li/rsc-advances

\section{Thiourea bridged periodic mesoporous organosilica with ultra-small Pd nanoparticles for coupling reactions $\uparrow$}

\author{
Esmail Doustkhah, abc Sadegh Rostamnia, (iD *a Masataka Imura, ${ }^{\mathrm{b}}$ Yusuke Ide, (D) ${ }^{\mathrm{b}}$

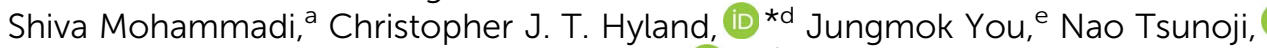 \\ Behzad Zeynizadeh ${ }^{c}$ and Yusuke Yamauchi (iD *egh
}

A synthesis of thiourea-bridged mesoporous organosilica is reported. Using transmission electron microscopy, low-angle XRD measurements and $\mathrm{N}_{2}$ adsorption-desorption isotherms the formation of a highly ordered mesoporous structure with a high surface area $\left(>400 \mathrm{~m}^{2} \mathrm{~g}^{-1}\right.$ ) has been confirmed. Deposition of $\mathrm{Pd}$ nanoparticles inside the mesopores resulted in a material that showed excellent catalytic activity in a Suzuki-coupling reaction and retained this activity over several consecutive runs.

\section{Introduction}

Within the field of mesoscale nanoarchitecturing, ${ }^{1}$ the creation of organic-inorganic hybrid mesoporous silica (HMS) materials is an attractive approach to prepare innovative materials with new functionalities. ${ }^{2}$ Many types of synthetic design strategies have been reported for the preparation of HMS materials with different pore sizes, mesostructures and framework compositions. ${ }^{3}$ To functionalize the pore walls of HMS with organic components, there are two typical approaches: postfunctionalization and co-condensation methods. In the postfunctionalization method, modification of the organic component is not uniform, and sometimes pore blocking and reduction in surface area occurs.

Synthesis of periodic mesoporous organosilicas (PMOs) can be accomplished by condensation of a bis- or trisorganosiloxane bridge with or without a silica precursor (e.g.,

${ }^{a}$ Department of Chemistry, Faculty of Science, University of Maragheh, Maragheh 55181-83111, Iran. E-mail: srostamnia@gmail.com

${ }^{b}$ International Center for Materials Nanoarchitechtonics (MANA), National Institute for Materials Science (NIMS), 1-1 Namiki, Tsukuba, Ibaraki 305-0044, Japan

'Department of Chemistry, Faculty of Science, Urmia University, Urmia 57159-165, Iran

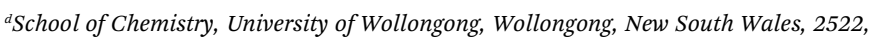
Australia.E-mail: chrhyl@uow.edu.au

${ }^{e}$ Department of Plant \& Environmental New Resources, Kyung Hee University, 1732 Deogyeong-daero, Giheung-gu, Yongin-si, Gyeonggi-do 446-701, South Korea

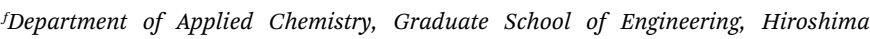
University, 1-4-1 Kagamiyama, Higashihiroshima, Hiroshima 739-8527, Japan

${ }^{g}$ Australian Institute for Innovative Materials (AIIM), University of Wollongong, Squires Way, North Wollongong, NSW 2500, Australia. E-mail: yusuke@uow.edu.au

${ }^{n}$ School of Chemical Engineering \& Australian Institute for Bioengineering and Nanotechnology (AIBN), The University of Queensland, Brisbane, QLD 4072, Australia $\dagger$ Electronic supplementary information (ESI) available. See DOI: $10.1039 / \mathrm{c} 7 \mathrm{ra} 11711 \mathrm{f}$
TEOS of TMOS) in the presence of a soft-template. ${ }^{4}$ These mesoporous organosilicas are expected to be applied to a vast variety of applications, including as photo-catalysts and as adsorbents. $^{5}$ Herein, we have synthesized a new thiourea bridged PMO (TU-PMO) using 1,3-bis(3-(triethoxysilyl)propyl) thiourea (bis-thiourea). In the case of the co-condensation method, it is well known that the mesostructural ordering seriously decreases when increasing the ratio of organosilica parts. However, our PMO shows a perfect 2D hexagonal mesostructure. The thiourea units are good at capturing metal nanoparticles due to effective interactions. ${ }^{6}$ Therefore, we can expect that the metal nanoparticles can be deposited only inside the mesopores, without uncontrollable external deposition. We have successfully deposited very small and uniform Pd nanoparticles inside the thiourea bridged PMO and demonstrated its ability to act as a catalyst in Suzuki cross-coupling reactions.

Our new mesoporous organosilica has a thiourea skeleton embedded within the pore walls, which is important for loading of Pd nanoparticles. Here, P123 was used as a soft-template and TEOS was also added for the reinforcement of the framework. The co-condensation reaction of TEOS and bis-thiourea was carried out in the presence of P123 under acidic conditions and the template was extracted by ethanol. For the Pd nanoparticle deposition inside the pores, the Pd species was introduced to the TU-PMO and then reduced using $\mathrm{NaBH}_{4}$ (Fig. 1).

\section{Experimental section}

Synthesis of 1,3-bis(3-(triethoxysilyl)propyl)thiourea (bisthiourea) bridge

First, $\mathrm{CS}_{2}$ (2 mmol) was added to (3-aminopropyl)triethoxysilane (APTES) (4 mmol) dropwise at room temperature and the mixture stirred for $3 \mathrm{~min}$ (Fig. S1 $\dagger$ ). Then, the temperature was raised to $100{ }^{\circ} \mathrm{C}$ under inert atmosphere and allowed to stir for 


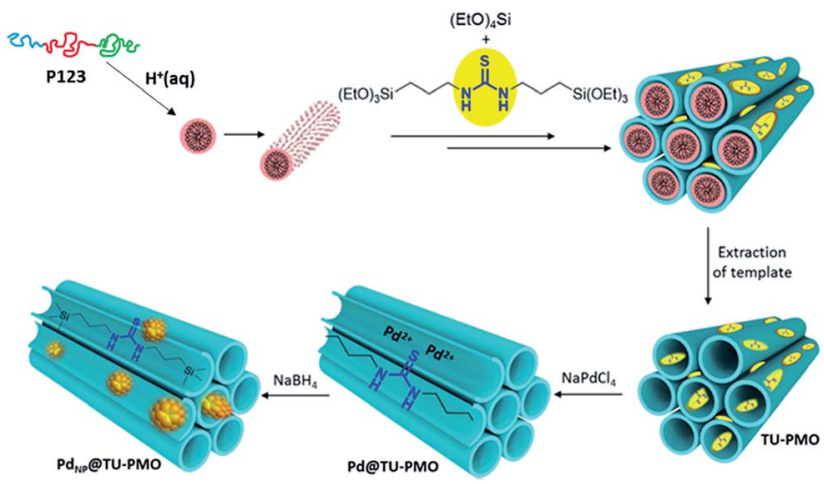

Fig. 1 General pathway for the synthesis of Pd@TU-PMO.

30 min. After complete evolution of $\mathrm{H}_{2} \mathrm{~S}$ gas from reaction mixture, indicating completion of the reaction, the mixture was cooled to room temperature and the final product was produced as a dark yellow liquid. This product was characterized by both ${ }^{1} \mathrm{H}$-NMR and ${ }^{13} \mathrm{C}$-NMR and was used as-is in the next step (Fig. S2 and S3†).

\section{Preparation of TU-PMO}

Typically, $2 \mathrm{~g}$ of P123 was dissolved in $75 \mathrm{~mL} \mathrm{HCl}_{(\mathrm{aq})}(2 \mathrm{M})$ and allowed to stir for several hours to obtain a clear solution. Then, the solution was warmed to $40{ }^{\circ} \mathrm{C}$ for $4 \mathrm{~h}$. After this time, a mixture of the bis-thiourea prepared above $(2 \mathrm{mmol})$ and TEOS (16 mmol, $3.7 \mathrm{~mL}$ ) was added to the solution drop-wise and the reaction allowed to stir for $24 \mathrm{~h}$. After this time, the reaction was stopped and aged at $100{ }^{\circ} \mathrm{C}$ with the same liquor for $24 \mathrm{~h}$. Afterwards, the resulting yellow solid was collected by filtration and extracted in a Soxhlet for 3 days with EtOH as an eluent. CHN analysis of TU-PMO was obtained and results showed that the percentage of organic loading is $19 \mathrm{wt} \%$.

\section{Synthesis of Pd@TU-PMO}

$\mathrm{NaPdCl}_{4}(0.10 \mathrm{~g})$ was dissolved in distilled water $(5 \mathrm{~mL})$ and this solution was added to a suspension of TU-PMO $(1 \mathrm{~g})$ in EtOH (20 $\mathrm{mL}$ ). After stirring for $1 \mathrm{~h}$ at $\mathrm{rt}$, the resulting solid was collected, washed with $\mathrm{EtOH}$ and dried in a vacuum oven for $60 \mathrm{~min}$ at $60^{\circ} \mathrm{C}$. The resulting solid was then dispersed in $\mathrm{MeOH}(20 \mathrm{~mL})$ and a solution of $\mathrm{NaBH}_{4}(19 \mathrm{mg})$ in $\mathrm{MeOH}(10 \mathrm{~mL})$ was added dropwise. After stirring for $30 \mathrm{~min}$ at room temperature, the final resulting solid was collected by filtration, washed and dried at $60{ }^{\circ} \mathrm{C}$ in vacuum oven. The final product was named Pd@TU-PMO.

\section{General procedure for Suzuki-Miyaura reactions}

In a typical procedure, an aryl halide $(2 \mathrm{mmol})$ and arylboronic acid $(2.1 \mathrm{mmol})$ were added to a mixture of $\mathrm{K}_{2} \mathrm{CO}_{3}(4 \mathrm{mmol})$ and Pd@TU-PMO (57 mg) in $5 \mathrm{~mL} \mathrm{H}_{2} \mathrm{O}$. The reactions were allowed to stir at room temperature and monitored by GC. The reaction conversion and product yield were also determined using GC with biphenyl as an internal standard. For the recycling study, after reaction completion, the reaction mixture was diluted and the product was extracted with dichloromethane. The catalyst was separated by centrifuge and carefully washed with dichloromethane. The catalyst was dried under vacuum at $70^{\circ} \mathrm{C}$ and then re-used in the cross-coupling reaction.

\section{Characterization}

Scanning electron microscopic (SEM) images were recorded on a Hitachi S-4800 filed emission scanning electron microscope (FE-SEM). Transmission electron microscopic (TEM) and elemental mapping images were taken by a JEM-2100F with an accelerating voltage of $200 \mathrm{kV}$. FT-IR spectra were obtained by a Shimadzu IR-460 spectrometer. After separation of the catalyst with centrifuge, the organic liquor was dried over $\mathrm{MgSO}_{4}$, evaporated in vacuo and the organic residue was (if necessary, it was purified by column chromatography) analyzed by ${ }^{1} \mathrm{H}$ and ${ }^{13} \mathrm{C}$ NMR. ${ }^{1} \mathrm{H}$ and ${ }^{13} \mathrm{C}$ NMR spectra were obtained on a JEOL Delta, $300 \mathrm{MHz}$ spectrometer. ${ }^{29} \mathrm{Si}$ magic-angle spinning (MAS) NMR spectra and ${ }^{13} \mathrm{C}$ cross-polarized (CP) MAS NMR spectra were recorded at $119.17 \mathrm{MHz}$ on a Varian 600PS solid-state NMR spectrometer using a $6 \mathrm{~mm}$ diameter zirconia rotor. Low-angle XRD patterns were collected on a Rigaku NANO-Viewer ( $\mathrm{Cu} \mathrm{K} \alpha)$. $\mathrm{N}_{2}$ adsorption-desorption isotherms were measured using a Quantachrome Autosorb at $77 \mathrm{~K}$. X-ray photoelectron spectroscopy (XPS) spectra were obtained on a 8025-BesTec twin anode XR3E2 X-ray source system at room temperature; all spectra were calibrated to $\mathrm{C} 1 \mathrm{~s}(285.0 \mathrm{eV})$ as a reference. The Pd loading amount was determined by inductively coupled plasma atomic emission spectroscopy (ICP-AES) on a Perkin Elmer 2100DV.

\section{Results and discussion}

After synthesis of Pd@TU-PMO, its Pd content was calculated to be $3.7 \mathrm{wt} \%$ according to ICP-AES analysis. XPS measurement was carried out for the Pd supported TU-PMO before and after reduction with $\mathrm{NaBH}_{4}$ (Fig. S4†). The presence of two characterized peaks at 337.9 and $334.1 \mathrm{eV}$ can be assigned to Pd in the +2 oxidation state before reduction with $\mathrm{NaBH}_{4}$. A small peak shift after reduction with $\mathrm{NaBH}_{4}$ shows that the Pd species has been reduced to its metallic form.

The $\mathrm{N}_{2}$ adsorption-desorption isotherm for TU-PMO shows a typical type IV isotherm, which has been seen in SBA-15 type mesoporous silica (Fig. 2a). The BET surface area was calculated to be $c a .410 \mathrm{~m}^{2} \mathrm{~g}^{-1}$ (Table S1†). Even after deposition of Pd nanoparticles, the high surface area still remained (Table S1 $\dagger$ ). The average pore sizes were estimated to be $c a .6 .1 \mathrm{~nm}$. Lowangle XRD patterns for TU-PMO shows one sharp peak at $2 \theta=0.92^{\circ}\left(d_{10}=9.60 \mathrm{~nm}\right)$, which originates from the periodicity of the mesoporous structure (Fig. 2b). After the Pd deposition, this peak was maintained and slightly shifted to higher degree $\left(2 \theta=0.95^{\circ}, d_{10}=9.30 \mathrm{~nm}\right)$. The framework of TU-PMO was characterized by FT-IR analysis (Fig. 2c). A broad and small band at $3265 \mathrm{~cm}^{-1}$ can be attributed to $\mathrm{N}-\mathrm{H}$ stretching and two sharp peaks at 2974 and $2928 \mathrm{~cm}^{-1}$ can be attributed to the asymmetric and symmetric stretching mode of aliphatic $\mathrm{C}-\mathrm{H}$ bonds in propyl chain, respectively. ${ }^{7}$ A small peak at $2887 \mathrm{~cm}^{-1}$ 

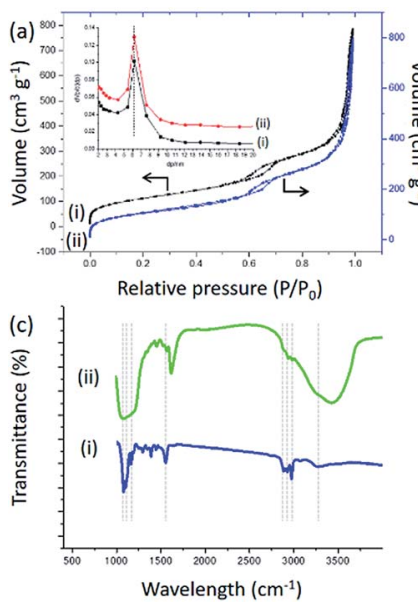

(d)
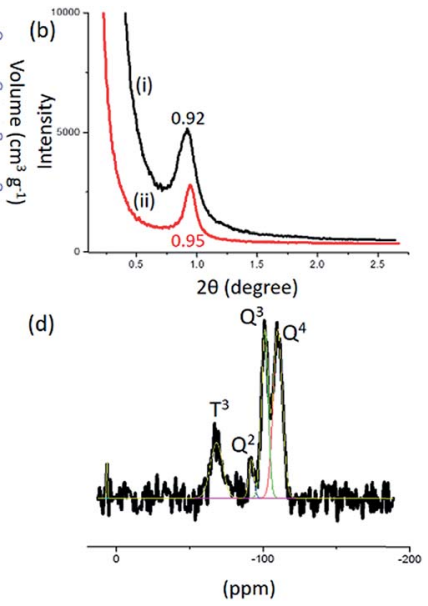

Fig. 2 (a) $\mathrm{N}_{2}$ adsorption-desorption isotherms and (b) low-angle XRD patterns for (i) TU-PMO and (ii) Pd@TU-PMO, (c) FT-IR for (i) bisthiourea and (ii) TU-PMO and, and (d) solid state ${ }^{29} \mathrm{Si} \mathrm{NMR} \mathrm{of} \mathrm{TU-PMO,}$ respectively. Characteristic peaks at 3265, 2974, 2928, 2887, 1553, 1167,1104 , and $1080 \mathrm{~cm}^{-1}$ are marked by dash lines.

corresponds to the aliphatic chain. Another peak at $1553 \mathrm{~cm}^{-1}$ can be assigned to $\mathrm{C}=\mathrm{S}$ bond stretching of the thiourea. A peak at $1167 \mathrm{~cm}^{-1}$ can be attributed to the stretching vibration mode of the $\mathrm{C}-\mathrm{N}$ bond. Two peaks at 1104 and $1080 \mathrm{~cm}^{-1}$ can be related to the $\mathrm{Si}-\mathrm{O}-\mathrm{Si}$ linkage.

Analysis of the solid state ${ }^{29}$ Si NMR provides further evidence for the structure of TU-PMO. A signal with a chemical shift of $-66.9 \mathrm{ppm}$ corresponds to the $\mathrm{T}^{3}\left((\mathrm{SiO})_{3} \mathrm{Si}-\mathrm{R}-\mathrm{Si}(\mathrm{OSi})_{3}\right)$ (Fig. 2d). ${ }^{8}$ The presence of this peak shows that the bis-thiourea bridge has been successfully attached to the mesoporous structure. Appearance of $\mathrm{T}^{3}$ indicates a type of Si in which one of the four bonds of $\mathrm{Si}$ is linked to a carbon atom. Additional peaks at $-110.4,-100.8$, and $-91.9 \mathrm{ppm}$ are attributed to the three different type $\mathrm{Si}$ atoms of $\mathrm{Q}^{4}\left[(-\mathrm{OSi})_{4} \mathrm{Si}\right], \mathrm{Q}^{3}\left[(-\mathrm{OSi})_{3} \mathrm{Si}-\mathrm{OH}\right]$, and $\mathrm{Q}^{2}\left[(-\mathrm{OSi})_{2} \mathrm{Si}(-\mathrm{OH})_{2}\right]$ in the TU-PMO structure. ${ }^{9}$ The area ratio of $\mathrm{Q}^{4}: \mathrm{Q}^{3}: \mathrm{Q}^{2}$ is $54: 41: 5$. Furthermore, the ratio of $\mathrm{T}: \mathrm{Q}$ is $13: 87$, which also shows the ratio of organosilicone to soley inorganic Si atoms. Solid-state ${ }^{13} \mathrm{C}$-NMR of TU-PMO shows obvious peaks at 11.6, 16.8, 25.4, 45.0, and $57.9 \mathrm{ppm}$, which can be assigned to the organic structure of the bis-thiourea. This provides important evidence that the organic bis-thiourea structure is well preserved after being embedded into the TUPMO structure (Fig. S5 $\dagger$ ). A decrease in the intensity of some signals can be attributed to the hydrolysis of triethoxysilyl groups of bis-thiourea. Also, disappearance of low intensity signals due to the silica matrix is expected.

Thermal gravimetry analysis (TGA) of TU-PMO can provide further evidence for the presence of the organic thiourea (Fig. S6 ${ }^{\circ}$ ). The weight loss, starting from $200^{\circ} \mathrm{C}$ to $500{ }^{\circ} \mathrm{C}$ can be attributed to the thiourea bridges of TU-PMO, which are approximately around $24 \mathrm{wt} \%$.

TEM and SEM observation of TU-PMO indicates that there are a large number of interwoven rods with widths around $100 \mathrm{~nm}$ (Fig. 3). Also, the original morphology was retained after deposition of Pd nanoparticles. From the TEM images of TU-

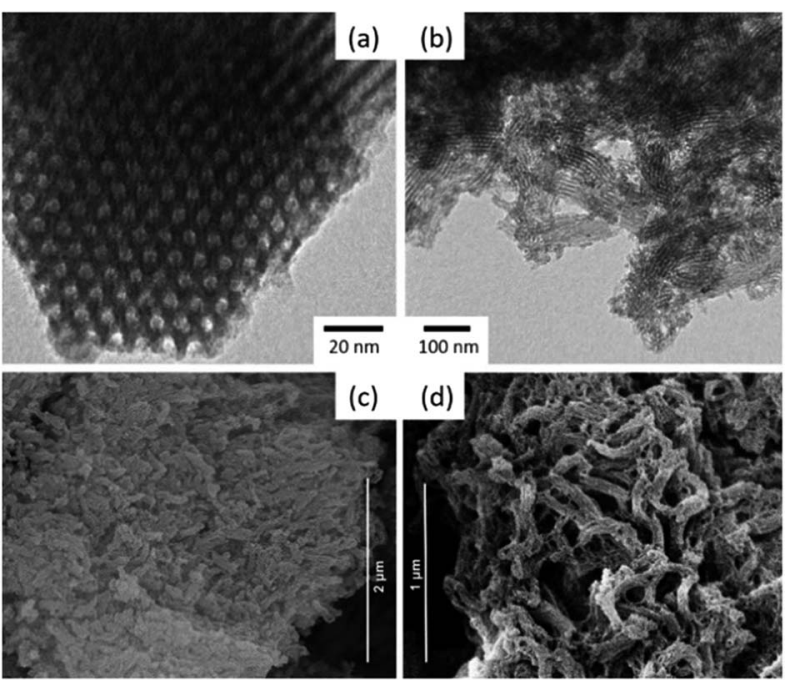

Fig. 3 (a, c) TEM and SEM images of TU-PMO, and (b, d) TEM and SEM images of Pd@TU-PMO.

PMO and Pd@TU-PMO, both honeycomb arrangement of mesopores and tubular mesochannels were confirmed (Fig. 3a and $\mathrm{b}$ ), indicating the formation of a $2 \mathrm{D}$ hexagonally ordered mesoporous structure. Although TEM image shows a high ordering of the 2D hexagonal mesostructure, low angle XRD patterns did not show (11) and (20) diffraction peaks. This is because the mesostructural domains are quite small, compared to normal SBA-15 material prepared using the P123 template. The pore-to-pore distance is around $11 \mathrm{~nm}$, which matches the calculated value from low-angle XRD pattern $\left(d_{10} \times 2 / \sqrt{ } 3=9.60 \mathrm{~nm} \times 2 / \sqrt{ } 3=11.1 \mathrm{~nm}\right)$. High-angle annular dark-field scanning transmission electron microscopy (HAADF-

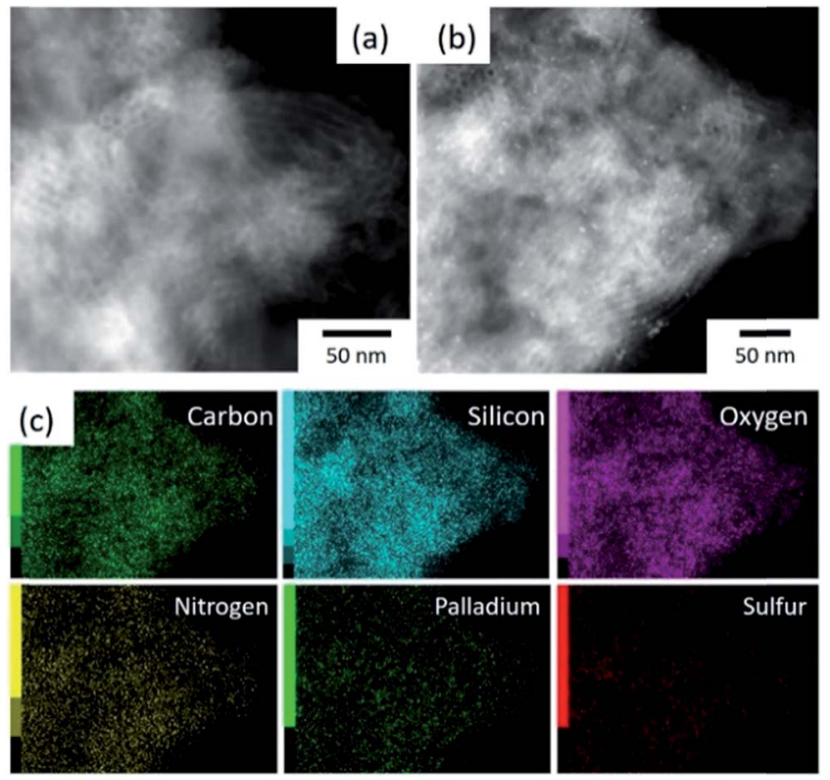

Fig. 4 (a) HAADF-STEM images of (a) TU-PMO and (b) PdaTU-PMO. (c) Elemental mapping images for carbon, silicon, oxygen, nitrogen, palladium, and sulfur of Pd@TU-PMO. 
STEM) image can visualize the presence of fine Pd nanoparticles (Fig. 4a and b). The hysteresis diagram of Pd nanoparticle size distribution was prepared from the HAADF-STEM image and revealed the average particle size as $1.8 \mathrm{~nm}$ - these small-sized Pd nanoparticles with a uniform size distribution were well dispersed throughout the structure (Fig. S7 $\dagger$ ). Elemental mapping images further support the formation of Pd located in the TU-PMO (Fig. 4c).

We tested the catalytic activity of Pd@TU-PMO in a model Suzuki-Miyaura cross-coupling reaction between 4-bromoacetophenone and phenylboronic acid. A screen of reaction solvents for this coupling quickly revealed DMF and water as the optimum solvents, providing the coupling product in $96 \%$ yield in each case (Fig. 5a). The remaining reaction parameters were investigated using water as a solvent, given its low cost and environmentally friendly nature. Increasing the temperature to $80{ }^{\circ} \mathrm{C}$ improved the yield of the reaction slightly to $100 \%$ (Fig. 5b). Finally, variation of catalyst amount revealed that $57 \mathrm{mg}$ of Pd@TU-PMO was optimum for $2 \mathrm{mmol}$ of $p$-bromoacetophenone (Fig. $5 \mathrm{c}$ ). Because the catalyst is heterogeneous, higher amounts may have an inverse effect on the catalytic efficiency, due to adsorption of the substrate onto the mesoporous structure. Monitoring of the reaction progress every $30 \mathrm{~min}$ revealed that the reaction was initially slow (probably
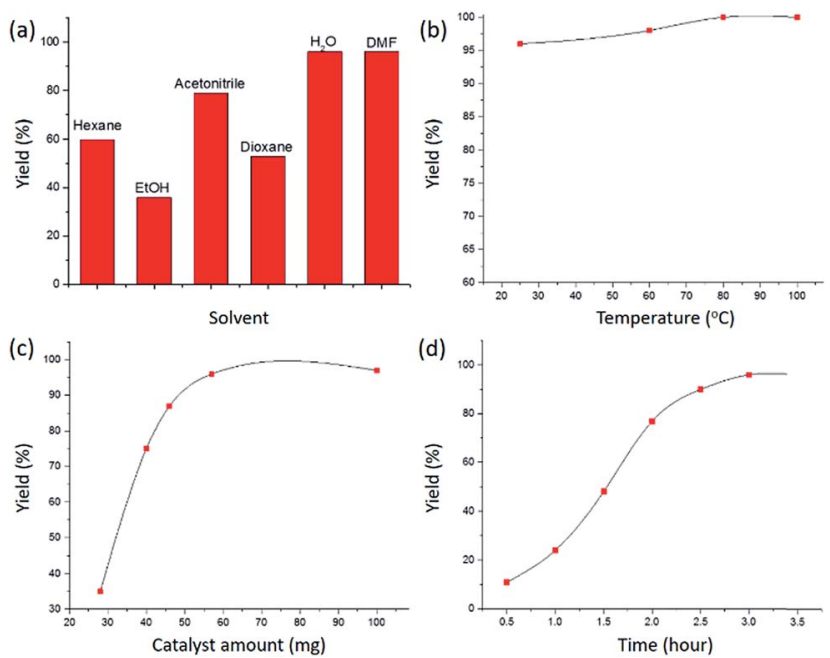

Fig. 5 (a) Solvent effect on the coupling of $p$-bromoacetophenone and phenylboronic acid (reaction conditions: $p$-bromoacetophenone (2 mmol), phenylboronic acid ( $2.1 \mathrm{mmol}), \mathrm{K}_{2} \mathrm{CO}_{3}(4 \mathrm{mmol})$, and catalyst (57 mg) in the presence of $5 \mathrm{~mL}$ solvent over 4 hours at room temperature). (b) The effect of temperature on the progress of Suzuki coupling reaction (reaction conditions: $p$-bromoacetophenone (2 $\mathrm{mmol})$, phenylboronic acid $(2.1 \mathrm{mmol}), \mathrm{K}_{2} \mathrm{CO}_{3}(4 \mathrm{mmol})$, and catalyst (57 mg) in the presence of $5 \mathrm{~mL} \mathrm{H}_{2} \mathrm{O}$ as a solvent over 4 hours, at various temperatures). (c) The effect of the catalytic amount on the progress of Suzuki coupling reaction (reaction conditions: $p$-bromoacetophenone (2 mmol), phenylboronic acid (2.1 mmol), $\mathrm{K}_{2} \mathrm{CO}_{3}(4$ $\mathrm{mmol}$ ) in the presence of $5 \mathrm{~mL} \mathrm{H}_{2} \mathrm{O}$ over 4 hours at room temperature). (d) Kinetic study on the progress of Suzuki coupling reaction (reaction conditions: $p$-bromoacetophenone $(2 \mathrm{mmol})$, phenylboronic acid $(2.1 \mathrm{mmol}), \mathrm{K}_{2} \mathrm{CO}_{3}(4 \mathrm{mmol})$, and catalyst $(57 \mathrm{mg})$ in the presence of $5 \mathrm{~mL} \mathrm{H} \mathrm{H}_{2} \mathrm{O}$, at room temperature).
Table 1 Synthesis of biaryl derivatives under the optimized conditions. In a typical procedure, aryl halide $(2 \mathrm{mmol})$, arylboronic acids (2.1

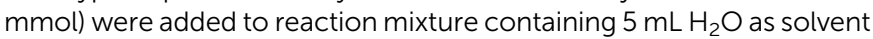
and $\mathrm{K}_{2} \mathrm{CO}_{3}(4 \mathrm{mmol})$ as base in the presence of PdaTU-PMO $(57 \mathrm{mg})$ over 4 hours. Then the reaction was carried out at room temperature. The reaction progress was monitored by GC and the final product yield was identified by GC, using biphenyl as an internal standard. NMR data for the coupling products are supplied in the ESI

\begin{tabular}{lllllll}
\hline & & & & & \\
\end{tabular}

due to an induction period), and that the rate increased until the reaction was complete at $3 \mathrm{~h}$ (Fig. $5 \mathrm{~d}$ ).

Following this optimization study, the generality of mesoporous Pd@TU-PMO towards Suzuki-coupling of a range of haloaryls with phenylboronic acid derivatives was studied. This study revealed that the catalyst was very active towards a diverse set of differentially substituted aryl bromides and aryl iodides, with each case providing good to excellent yields (Table 1). The recyclability of the Pd@TU-PMO catalyst was also investigated and gratifyingly it was found that the catalyst could be re-used over several consecutive cycles with minimal loss of catalytic activity (Fig. S8†).

\section{Conclusion}

We have synthesized a thiourea-based PMO to produce ultrasmall Pd nanoparticles inside the mesopores. We then demonstrated that our PMO is a promising catalyst for Suzuki cross-coupling reactions with high efficiency and recyclability. The thiourea organosiloxane bridge used in this study has the potential to be used in the synthesis of other hybrid nanostructures. Furthermore, the synthesized PMOs can be used for immobilization of other types of catalytically active metal 
nanoparticles besides Pd. Also, TU-PMO itself, without any additional post-modification, may potentially be used as a solid $\mathrm{H}$-bonding organocatalyst owing to the presence of the thiourea groups.

\section{Conflicts of interest}

There are no conflicts to declare.

\section{Notes and references}

1 (a) R. R. Salunkhe, Y. V. Kaneti, J. Kim, J. H. Kim and Y. Yamauchi, Acc. Chem. Res., 2016, 49, 2796; (b) J. Tang and Y. Yamauchi, Nat. Chem., 2016, 8, 638.

2 (a) S. Rostamnia and E. Doustkhah, RSC Adv., 2014, 4, 28238; (b) S. Fujita, M. P. Kapoor and S. Inagaki, Organic-Inorganic Hybrid Mesoporous Silica, in Nanohybridization of OrganicInorganic Materials. Advances in Materials Research, ed. A. Muramatsu and T. Miyashita, Springer, Berlin, Heidelberg, vol. 13, 2009.

3 Q. Yang, J. Liu, L. Zhang and C. Li, J. Mater. Chem., 2009, 19, 1945.

4 (a) S. Inagaki, S. Guan, Y. Fukushima, T. Ohsuna and O. Terasaki, J. Am. Chem. Soc., 1999, 121, 9611; (b) S. Inagaki, S. Guan, T. Ohsuna and O. Terasaki, Nature, 2002, 416, 304; (c) S. Rostamnia, E. Doustkhah, R. Bulgar and B. Zeynizadeh, Microporous Mesoporous Mater., 2016, 225, 272.

5 (a) M. Ohashi, M. Aoki, K. Yamanaka, K. Nakajima, T. Ohsuna, T. Tani and S. Inagaki, Chem.-Eur. J., 2009, 15, 13041; (b) M. I. López, D. Esquivel, C. Jiménez-Sanchidrián, P. Van Der Voort and F. J. Romero-Salguero, J. Phys. Chem. $C$, 2014, 118, 17862; (c) N. Mizoshita, T. Tani and S. Inagaki, Chem. Soc. Rev., 2011, 40, 789; (d) B. Karimi, D. Elhamifar, J. H. Clark and A. J. Hunt, Chem.-Eur. J., 2010, 16, 8047; (e) S. S. Park, M. S. Moorthy and C. S. Ha, NPG Asia Mater., 2014, 6, e96.

6 (a) A. S. Al-Kady, M. Gaber, M. M. Hussein and E. M. Ebeid, J. Phys. Chem. A, 2009, 113, 9474; (b) T. Mandal, V. Stavila, I. Rusakova, S. Ghosh and K. H. Whitmire, Chem. Mater., 2009, 21, 5617-5626.

7 (a) Y. Niu, H. Liu, R. Qu, S. Liang, H. Chen, C. Sun and Y. Cui, Ind. Eng. Chem. Res., 2015, 54, 1656; (b) Y. Zhao, R. Jin, Y. Chou, Y. Li, J. Lin and G. Liu, RSC Adv., 2017, 7, 22592; (c) E. Doustkhah, S. Rostamnia, H. G. Hossieni and R. Luque, ChemistrySelect, 2017, 2, 329; (d) N. Mizoshita, M. Ikai, T. Tani and S. Inagaki, J. Am. Chem. Soc., 2009, 131, 14225.

8 D. Chandra, T. Yokoi, T. Tatsumi and A. Bhaumik, Chem. Mater., 2007, 19, 5347.

9 T. Tokunaga, M. Shoiriki, T. Mizumo and Y. Kaneko, J. Mater. Chem. C, 2014, 2, 2496. 